\title{
PinX1 the tail on the chromosome
}

\author{
F. Brad Johnson
}

Department of Pathology and Laboratory Medicine, University of Pennsylvania School of Medicine, Philadelphia, Pennsylvania, USA.

\begin{abstract}
The PinX1 protein inhibits telomerase, an enzyme that lengthens telomeres the structures that protect the ends of chromosomes. Loss of PinX1 leads to increased telomere length along with defects in chromosome dynamics. In this issue of the JCI, Zhou et al. present novel evidence from human tumors and mouse models indicating that PinX1 is a clinically significant tumor suppressor. Importantly, the genome-destabilizing effects of PinX1 loss appear to depend on telomerase activity, raising new models and questions for how telomeres and telomerase contribute to the development of cancer.
\end{abstract}

\section{Telomeres and telomerase}

Telomeres cap the ends of chromosomes, concealing them from the checkpoint responses that recognize broken DNA ends and protecting them from inappropriate exonucleolytic attack and recombination (1). Telomeres are thus essential to chromosome stability. However, for several reasons, telomeres shorten with each round of DNA replication and can eventually become uncapped by this attrition, leading to their recognition as DNA breaks and thus to permanent cell-cycle arrest (cell senescence) or apoptosis.

Telomere shortening can be slowed or reversed by the enzyme telomerase, which at its core contains a catalytic subunit, telomerase reverse transcriptase (TERT), and an associated RNA template, telomerase RNA component (TERC), which codes for the telomere repeat. However, in most somatic cells, telomerase is present at levels that are insufficient to prevent telomere shortening with cell division and advancing age. In addition to its role at telomeres, evidence has mounted that telomerase may also have "extracurricular" activities, including functions in mitochondria, DNA repair, stem cell maintenance, and modulation of Wnt signaling (2-5). The last activity (and possibly the others) apparently requires TERT but not TERC and is thus independent of classical telomerase activity.

In addition to telomerase, a large number of factors help maintain telomere integrity. In mammals, chief among these are the members of the shelterin complex, including the duplex telomere repeat bind-

Conflict of interest: The author has declared that no conflict of interest exists.

Citation for this article: J Clin Invest. 2011; 121(4):1242-1244. doi:10.1172/JCI57024. ing proteins TRF1 and TRF2 (1). Shelterin proteins recruit a host of other factors to the telomere including PinX1. TRF1 binds PinX1, which directly interacts with and inhibits telomerase action (6).

\section{Telomere biology and cancer}

Telomeres and telomerase influence cancer in several ways (3). It is thought that the shortening of telomeres in human cells provides a brake to the unlimited cell division characteristic of cancers. Telomere shortening and eventual uncapping can thus be considered a type of clock, whose alarm is set off when cells have undergone more divisions than would be expected within a natural human lifespan - an indication that the proliferative controls of such cells have gone awry and that division should be stopped. There is abundant evidence supporting this idea. For example, the key tumor suppressors p53 and Rb respond to uncapped telomeres by limiting cell proliferation or survival, and premalignant lesions contain cells with very short telomeres and that have become senescent. However, although telomere length-based barriers likely provide net inhibition of cancer progression, the rare cells that evade these barriers (e.g., via compromised p53 and Rb pathways) will incur the consequences of dysfunctional telomeres. For example, these can include chromosome end-to-end fusions, leading to dicentric chromosomes and yielding aneuploidy or chromosome rearrangements. The eventual activation of a telomere maintenance mechanism, typically taking the form of elevated expression of telomerase, in these incipient cancer cells can then support further cell division. In addition, whereas senescence caused by telomere uncapping limits carcinogenesis in a cell-autonomous fashion, senescent cells produce tumor- promoting factors that may abet cancers derived from neighboring cells (7). Thus, telomere shortening can have both positive and negative effects on carcinogenesis.

A cytogenetic characteristic of epithelial cancers (i.e., carcinomas) is frequent and complex nonreciprocal translocations. This contrasts with the comparatively stable genomes of nonepithelial cancers. Although the basis of cytogenetic instability in carcinoma is not fully understood, a leading candidate is chromosome endfusion caused by uncapped telomeres (8). Telomere dysfunction may thus drive the development of carcinomas more than it drives nonepithelial cancers. Consistent with this idea, telomere shortening in $p 53^{+/-}$ $m \mathrm{Terc}^{-1-}$ mice shifts the spectrum of tumors observed in $p 53^{+/-}$mice from lymphoma and sarcoma toward carcinoma (9).

\section{PinX1 is a haploinsufficient tumor suppressor}

PinX1 is encoded within the chromosome 8p23 region, which is frequently affected by loss of heterozygosity in human carcinomas (e.g., ref. 10). In this issue of the JCI, Zhou et al. report on their investigation of the possibility that PinX1 may be a tumor suppressor (11). Consistent with this idea, they found that PinX1 protein levels are generally reduced in breast cancer tissues and cell lines, lending support to earlier reports of lower levels in some other carcinomas including liver and gastric tumors. Next they generated mice lacking one copy of PinX1, which yielded reduced levels of PinX1 protein, two-fold increased telomerase activity, and an approximately $50 \%$ increase in telomere lengths in embryonic fibroblasts (MEFs) and adult tissues. Strikingly, the heterozygous mice developed increased rates of cancer, with a shift in tumor spectrum away from the lymphomas and sarcomas typical of mice and toward epithelial cancers more frequently seen in humans, including lung, liver, mammary, and gastrointestinal carcinomas. This shift also occurred when one or both copies of p53 were inactivated. Furthermore, the tumors retained the intact copy of PinX1. These data indicate that PinX1 is a haploinsufficient tumor suppressor. 


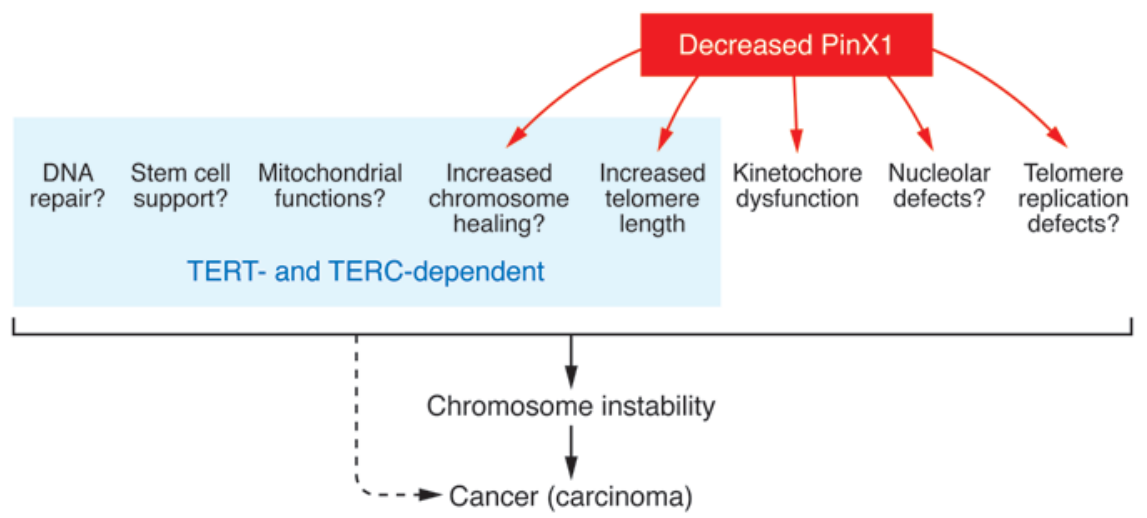

Figure 1

Consequences of insufficient PinX1 that may contribute to chromosome instability and cancer. The chromosome instability observed in cultured $\mathrm{Pin} X 1^{+/-}$MEFs required both TERT and TERC components of telomerase, and so the processes that are known or suspected to require TERT and TERC are indicated. The chromosome instability, and presumably cancer, in Pin $X 1^{+/-}$mice is due to a single process, or some combination of processes, that depend on telomerase and are sensitive to PinX1 dosage. However, the key processes are not known, and processes not yet defined could be involved. Speculative or uncertain aspects of the figure are indicated by question marks.

The mechanisms underlying cancer in PinX $1^{+/-}$mice may involve increased genome instability, because with passage in culture PinX1 $1^{+/-}$MEFs developed anaphase bridges, aneuploidy, and chromosome rearrangements, including nonreciprocal translocations. Similar instability was observed in the tumors from these mice. Importantly, genome instability in MEFs required both TERT and TERC, suggesting an important role for telomerase activity in this phenotype. Also of note, telomere dysfunction-induced foci (TIF) assays indicated no apparent defects in telomere capping.

\section{How does diminished PinX1 function drive chromosome instability and cancer?}

Beyond suggesting that PinX1 is an important tumor suppressor, the new findings may have uncovered a novel dimension of the interplay of telomere biology and cancer. A key question is how PinX1 deficiency leads to chromosome instability (Figure 1). In addition to regulating telomerase, PinX1 localizes to kinetochores (12) and to nucleoli (13), but whereas defects at these sites certainly might have an impact on genome stability and nucleolar PinX1 may regulate telomerase biogenesis (14), it is not clear how telomerase would act directly at these sites to drive genome instability. Zhou et al. instead suggest that long telomeres may themselves induce instability, an idea consistent with the requirement for telomerase activity. This is a fascinating suggestion, and one can imagine that excessive tracts of telomere repeats might destabilize chromosomes, for example, by being especially prone to replication or recombination abnormalities, by outstripping the capacity of natural levels of shelterin proteins to protect them, or possibly by soaking up factors that normally function at other genomic loci. On the other hand, TIF assays did not reveal uncapped telomeres in the PinX $1^{+/-}$cells, and so if there are defects in telomere capping, they must be relatively subtle or infrequent. Furthermore, previous studies have not provided any clear indications that long telomeres per se contribute directly to genome instability. For example, forced expression of telomerase in cultured normal human fibroblasts, although it lengthens telomeres, has only a beneficial effect on chromosome stability (15). In yeast, artificial lengthening of a telomere can lead to elevated levels of recombination-based telomere shortening, but this occurs only when a subset of the telomeres is lengthened, indicating it is not an intrinsic property of long telomeres (16). Furthermore, Zhou et al. suggest the mechanisms underlying the preferential increases in carcinomas observed in $\mathrm{PinX} 1^{+/-}$mice might be related to those driving similar tumor spectra following telomerase overexpression and telomere lengthening in K5-mTert (where K5 indicates Keratin 5) transgenic mice or following telomere shortening in $m \operatorname{Terc}^{-1-} p 53^{+/-}$mice $(9,17)$. However, the $K 5$ promoter is expressed principally in epithelia, which might explain the tumor spectrum of the K5-mTert mice; and carcinomas develop in $\mathrm{PinX} 1^{+/-}$mice regardless of p53 status, whereas $m$ Terc $^{-1-}$ mice with intact p53 or homozygous p53 inactivation do not develop the striking carcinomas observed in $m \mathrm{Terc}^{-/-} p 53^{+/-}$mice. Thus carcinomas may arise via distinct mechanisms in these different models. Also of note, the dual requirement for TERT and TERC for the chromosome instability of PinX1 $1^{+/}$cells suggests TERC-independent extracurricular TERT activities are unlikely to be involved.

How else might PinX1 deficiency lead to genome instability? Just as a blindfolded child playing the party game of pin the tail on the donkey sometimes misses the mark, telomerase can mistakenly append telomere repeats onto the termini of a broken chromosome, an event known as chromosome healing (18). These events are rare in normal cells but might be disinhibited by PinX1 deficiency, similar to healing of broken chromosomes in yeast lacking the Pif1 protein, which normally inhibits telomerase action at telomeres and DNA breaks (19). Although healing can stabilize the broken end (at least temporarily), it interferes with other repair mechanisms that would otherwise restore the chromosome. The acentric fragment would be lost and the new terminus bearing short telomere repeats might recombine with interstitial telomere repeats to generate translocations. It would be interesting to examine the translocation breakpoints in PinX $1^{+/-}$cells to test this speculative idea. Another possibility is that telomerase plays an indirect permissive role that cooperates with defects caused by reduced PinX1 levels to allow survival of cells with chromosome instability. These PinX1-related defects could be at nontelomeric sites, e.g., at kinetochores causing mitotic segregation defects or at the telomere. TRF1 has been found to facilitate telomere replication (20), and it is conceivable PinX1 cooperates with TRF1 in this capacity. In these scenarios, the permissive role of telomerase could be at telomeres or possibly at other targets of telomerase such as mitochondria. In this later case, the longer telomeres of $\mathrm{PinX} 1^{+/-}$ cells would merely correlate with, rather than contribute to, the instability.

\section{Additional questions}

The new findings raise additional questions. For example, at what stages of carcinogenesis are PinX1 levels decreased, and how does this timing correlate with 
the onset of chromosome instability? How might the status of PinX 1 in tumors guide therapy? The authors suggest that telomerase inhibitors may be useful for treating cancers lacking PinX1 function. This is an intriguing idea, provided the benefit to cancer cells of PinX1 loss persists in established tumors (e.g., via promoting telomere lengthening); however, if the key benefit occurs early, via enhanced genome instability, PinX1 status may not affect the sensitivity of mature tumors to telomerase inhibition. Fortunately, existing tools can be combined to address whether cancer in PinX1 $1^{+/-}$mice or the growth of PinX1-deficient tumor cell lines is particularly susceptible to telomerase inhibition, and so answers should soon be forthcoming.

\section{Acknowledgments}

The author thanks P. Lieberman, Y. Tzfati, R. Greenberg, and members of the Johnson lab for helpful discussions. This work was supported by NIH grant AG021521.

Address correspondence to: F. Brad Johnson, Department of Pathology and Laboratory Medicine, University of Pennsylvania
School of Medicine, 422 Curie Boulevard, Philadelphia, Pennsylvania 19104, USA. Phone: 215.573.5057; Fax: 215.573.6317; E-mail: johnsonb@mail.med.upenn.edu.

1. Palm W, de Lange T. How shelterin protects mammalian telomeres. Annu Rev Genet. 2008;42:301-334.

2. Kovalenko OA, et al. A mutant telomerase defective in nuclear-cytoplasmic shuttling fails to immortalize cells and is associated with mitochondrial dysfunction. Aging Cell. 2010;9(2):203-219.

3. Artandi SE, DePinho RA. Telomeres and telomerase in cancer. Carcinogenesis. 2010;31(1):9-18.

4. Park JI, et al. Telomerase modulates Wnt signalling by association with target gene chromatin. Nature. 2009; 460(7251):66-72.

5. Flores I, Benetti R, Blasco MA. Telomerase regulation and stem cell behaviour. Curr Opin Cell Biol. 2006;18(3):254-260.

6. Soohoo CY, Shi R, Lee TH, Huang P, Lu KP, Zhou $X Z$. Telomerase inhibitor PinX1 provides a link between TRF1 and telomerase to prevent telomere elongation.J Biol Chem. 2011;286(5):3894-3906.

7. Davalos AR, Coppe JP, Campisi J, Desprez PY. Senescent cells as a source of inflammatory factors for tumor progression. Cancer Metastasis Rev. 2010;29(2):273-283.

8. Thompson SL, Compton DA. Chromosomes and cancer cells [published online ahead of print December 29, 2010]. Chromosome Res. doi:10.1007/ s10577-010-9179-y.

9. Artandi SE, et al. Telomere dysfunction promotes non-reciprocal translocations and epithelial cancers in mice. Nature. 2000;406(6796):641-645.

10. Emi M, et al. Frequent loss of heterozygosity for loci on chromosome $8 \mathrm{p}$ in hepatocellular carcinoma, colorectal cancer, and lung cancer. Cancer Res. 1992;52(19):5368-5372.

11. Zhou XZ, et al. The telomerase inhibitor PinX1 is a major haploinsufficient tumor suppressor essential for chromosome stability in mice. J Clin Invest. 2011;121(4):1266-1282.

12. Yuan $\mathrm{K}$, et al. PinX1 is a novel microtubule-binding protein essential for accurate chromosome segregation. J Biol Chem. 2009;284(34):23072-23082.

13. Yoo JE, Oh BK, Park YN. Human PinX1 mediates TRF1 accumulation in nucleolus and enhances TRF1 binding to telomeres. J Mol Biol. 2009; 388(5):928-940.

14. Lin J, Blackburn EH. Nucleolar protein PinX1p regulates telomerase by sequestering its protein catalytic subunit in an inactive complex lacking telomerase RNA. Genes Dev. 2004;18(4):387-396.

15. Morales CP, et al. Absence of cancer-associated changes in human fibroblasts immortalized with telomerase. Nat Genet. 1999;21(1):115-118.

16. Li B, Lustig AJ. A novel mechanism for telomere size control in Saccharomyces cerevisiae. Genes Dev. 1996;10(11):1310-1326.

17. Gonzalez-Suarez E, Flores JM, Blasco MA. Cooperation between $\mathrm{p} 53$ mutation and high telomerase transgenic expression in spontaneous cancer development. Mol Cell Biol. 2002;22(20):7291-7301.

18. Gao Q, et al. Telomerase-dependent and -independent chromosome healing in mouse embryonic stem cells. DNA Repair (Amst). 2008;7(8):1233-1249.

19. Boule JB, Zakian VA. Roles of Pif1-like helicases in the maintenance of genomic stability. Nucleic Acids Res. 2006;34(15):4147-4153

20. Sfeir A, et al. Mammalian telomeres resemble fragile sites and require TRF1 for efficient replication. Cell. 2009;138(1):90-103.

\title{
The adaptive stroma joining the antiangiogenic resistance front
}

\author{
Oriol Casanovas
}

Tumor Angiogenesis Group, Translational Research Laboratory, Catalan Institute of Oncology — IDIBELL, L'Hospitalet de Llobregat, Spain.

\begin{abstract}
Resistance to antiangiogenic therapies in cancer involves both tumor cells and stromal components, but their relative contributions differ in each cancer subtype. In this issue of the JCI, Cascone et al. describe a stromal adaptation to antiangiogenic therapy in non-small cell lung carcinoma (NSCLC) models that include EGFR-driven vascular remodeling promoting resistance to VEGF inhibition. Their results suggest that the added benefit of dual VEGF/R and EGFR targeting in these models could be clinically relevant to fight resistance in NSCLC patients.
\end{abstract}

The growth of a tumor depends on vascular remodeling to ensure a continuous supply of nutrients and oxygen, and blockade of the formation of new blood vessels with antiangiogenic drugs is currently used to treat certain types of cancer. For its central role in promoting angiogenesis, VEGF is

Conflict of interest: The author has declared that no conflict of interest exists.

Citation for this article: J Clin Invest. 2011;

121(4):1244-1247. doi:10.1172/JCI46430. the main target of the currently approved antiangiogenic drugs. Nevertheless, clinical results demonstrate only moderate gains in time to progression and scarce benefits in overall survival, despite long-term treatment (1). Why are there such modest and short-lasting benefits of antiangiogenic therapies in the clinic? The initial hypothesis was that antiangiogenic therapy would not induce resistance (i.e., "resistant to resistance") because it targeted endothelial cells instead of the tumor cell itself (2). Never- theless, clinical and experimental evidence has been mounting that resistance to antiangiogenic therapy does indeed occur (3).

Among the tumor responses to therapy, it is critical to distinguish between refractoriness, sometimes called intrinsic resistance (4), and acquired resistance. Tumors have long been shown to have remarkable plasticity and adaptability to classical chemotherapy and radiation, and this plasticity contributes to evasion from antiangiogenic therapy (5-7). However, the specific mechanisms of acquired resistance to antiangiogenic therapies are unique, and may reverse after antiangiogenic therapy has been stopped (M. Pàez-Ribes and O. Casanovas, unpublished observations). This suggests that these forms of resistance may reflect adaptations to therapy rather than the mutations or gene amplifications that characterize acquired resistance to other thera- 